\title{
Energy dependence of crystal assisted extraction at the CERN SPS
}

\author{
G. Arduini, K. Elsener, G. Fidecaro, M. Gyr, W. Herr, J. Klem*, \\ U. Mikkelsen and E. Weisse (CERN, Geneva, Switzerland)
}

\section{ABSTRACT}

The feasibility of extracting particles from the halo of a circulating proton beam has been successfully demonstrated in an experiment at the SPS. Although most measurements were obtained at an intermediate energy of $120 \mathrm{GeV}$, more recently we had the opportunity to extract protons from a coasting beam at $14 \mathrm{GeV}$ and $270 \mathrm{GeV}$, thus exploiting the full available energy range of the SPS. In this report we present a comparison of the measurements at the three energies and a qualitative comparison with the predictions.

\section{INTRODUCTION}

Beam extraction with a bent crystal depends on parameters that change with the beam energy. Beam diffusion and the resulting impact parameters and angles depend on the beam energy and on the excitation that is used to make the beam particles hit the crystal. When the particles enter the crystal, their interactions are energy dependent. Multiple scattering, critical angle of channeling, and dechanneling in straight and bent parts of the crystal change as a function of beam energy. Consequently, the extraction efficiency for a given extraction set-up depends strongly on the energy of the particles. In the SPS we had the unique opportunity to extract protons at three beam energies: $14 \mathrm{GeV}, 120 \mathrm{GeV}$ and $270 \mathrm{GeV}$. For all energies a beam could be extracted with a good signal to background ratio and extraction efficiencies, extracted beam sizes and angular scan widths could be measured. The crystal properties such as length, thickness and bending radius were designed and originally optimized for an energy of $120 \mathrm{GeV}$ [1] and significant effects are expected for the large energy range available to us.

\section{ENERGY DEPENDENCE}

\subsection{Diffusion process}

The impact parameter and angle of a particle intercepted by the crystal depend on the emittance growth of the transverse betatron motion in the halo of the circulating beam. The natural diffusion in the SPS is very slow and an artificial amplitude growth using transverse noise was applied. In our experiment random, transverse kicks were provided by voltage noise between two electrostatic plates [2]. When the noise is uncorrelated and not synchronous with the beam, the average emittance growth per second

* Also: Helsinki Univ. of Technology, Finland can be expressed as:

$$
\frac{<\Delta \epsilon>}{\sec }=\frac{\beta \nu_{r e v} l^{2}}{2 d^{2}} \cdot \frac{<U^{2}>}{\gamma^{2} E_{0}^{2}}
$$

where $\beta$ is the transverse beta function at the noise source and $\nu_{\text {rev }}$ the revolution frequency. Further, $\left\langle U^{2}\right\rangle$ is the applied r.m.s. voltage squared between the plates of length $l$ with a distance $d$ between the plates. $\gamma$ is the relativistic factor and $E_{0}$ the proton rest energy. The predicted emittance growth was confirmed by measurements and scales with the energy squared. It has been shown in simulation [2] that the average impact parameter scales approximately linearly with the applied kick strength, and therefore decreases with the beam energy. It is difficult to obtain a fast emittance growth and large impact parameters for the highest energy of $270 \mathrm{GeV}$.

\subsection{Channeling properties}

\subsubsection{Critical angle}

The critical angle scales as $\frac{1}{\sqrt{p}}$ with the momentum $p$ and we find $39.8 \mu \mathrm{rad}$ for $14 \mathrm{GeV} / \mathrm{c}, 13.8 \mu \mathrm{rad}$ for $120 \mathrm{GeV} / \mathrm{c}$, and $9.1 \mu \mathrm{rad}$ for $270 \mathrm{GeV} / \mathrm{c}$ protons in the case of planar channeling in the (110) plane of silicon.

\subsubsection{Multiple scattering and multipass extraction}

When a proton passes the crystal in the amorphous region or is dechanneled, it experiences multiple scattering and is deflected by a random angle $\theta$ with a Gaussian distribution with zero mean value and a width:

$$
\theta=\frac{13.6 \mathrm{MeV}}{\beta c p} \sqrt{\frac{L_{e f f}}{X_{0}}}\left(1+0.038 \ln \left(\frac{L_{e f f}}{X_{0}}\right)\right)
$$

where $c p=120 \mathrm{GeV}, \beta \sim 1$, and $X_{0}$ is the radiation length of the crystal ( $\sim 9.4 \mathrm{~cm}$ for silicon). For a particle traversing the full crystal length of $4 \mathrm{~cm}$ this angle $\theta$ is approximately $66.5 \mu \mathrm{rad}$ at $120 \mathrm{GeV} / \mathrm{c}$ but usually the particles which undergo multiple scattering do not cross the full crystal length but only a small part of it (i.e. $L_{e f f}$, c.f. [3]). It has been shown [4] that the multi pass extraction process plays an important role for extraction from the SPS, i.e. particles traverse the crystal multiple times before they are eventually channeled. The multiple scattering experienced in such a passage determines the impact parameters for further intercepts on the crystal [3]: strong scattering leads to large impact parameters. Due to the strong dependence of the multiple scattering angle on the energy one can expect a strongly changed pattern of impact properties on later encounters of the proton on the crystal. It is also 
expected that the width of the vertical profiles of channeled particles is increased for lower energies.

\subsubsection{Dechanneling}

To be successfully extracted a particle must remain channeled, but interactions with electrons inside the crystal or with the nuclei in the lattice, can lead to dechanneling of initially channeled particles. This dechanneling processes can be approximated by an exponential decrease of the number of channeled particles: $n=n_{0} e^{-l / l_{0}}$ where $l_{0}$, called normal dechanneling length, is equal to $10.8 \mathrm{~cm}$ for [110] silicon planes and $120 \mathrm{GeV} / \mathrm{c}$ protons. This parameter scales as $1 / p$ with the momentum and becomes a very important factor at $14 \mathrm{GeV} / \mathrm{c}$, leading to a large dechanneling probability $\mathrm{P}_{d c}$. The dechanneling length for $14 \mathrm{GeV} / \mathrm{c}$ is only about $1.26 \mathrm{~cm}$, i.e. much smaller than the crystal length and we have to expect that more than $90 \%$ of the channeled particles dechannel within the crystal length. The extraction efficiency should therefore be significantly lower than for $120 \mathrm{GeV} / \mathrm{c}$.

\section{PREPARATION OF THE SPS}

The details of the experiment and the analysis can be found elsewhere $[1,5,6]$. However, a few particularities setting up the SPS for three significantly different energies to get comparable results, deserve some attention. The proton beam is injected into the SPS at a momentum of $14 \mathrm{GeV} / \mathrm{c}$ in two batches, one 1.2 seconds after the other, each $10 \mu \mathrm{s}$ long. Typical tunes during the whole cycle (flat bottom, acceleration and coast) are 26.62 and 26.58 in the horizontal and vertical plane, respectively. Injection oscillations and low frequency instabilities (resistive wall instabilities) are damped by a transverse feedback. A single batch (a few $10^{12}$ protons) was accelerated (except for the $14 \mathrm{GeV}$ coast) to the target energy. The functions of all the ring power converters were frozen at that point and the injection and the beam dump kickers were inhibited. The damper was active at injection and it was automatically deactivated after a few seconds. In order to damp the resistive wall instabilities during the coast at $14 \mathrm{GeV}$ and therefore guarantee a sufficient lifetime to make the extraction measurement possible, the strength of the octupoles for Landau damping had to be strongly increased (by a factor 2 radially and 5 vertically) as compared to that used for the high intensity fixed target beam ( $4.5 \times 10^{13}$ protons) at injection. After the initial adjustment of the orbit the RF frequency was switched off and a lifetime of about 4 hours (without crystal) was achieved at $14 \mathrm{GeV}$. The use of transverse noise or of adequate sextupoles, required for the extraction at higher energy, was not necessary at $14 \mathrm{GeV}$ because of the natural diffusion due to the strong octupolar component and to the spontaneous beam excitation.

\section{RESULTS}

The experimental procedure such as the alignment of the crystal and details about the angular scans are found in previous reports $[1,5]$. Angular scans are performed to align the crystal with the beam envelope and the results of three typical scans for the three energies are shown in Figs. 1 to 3.

\subsection{Angular scans and profiles}
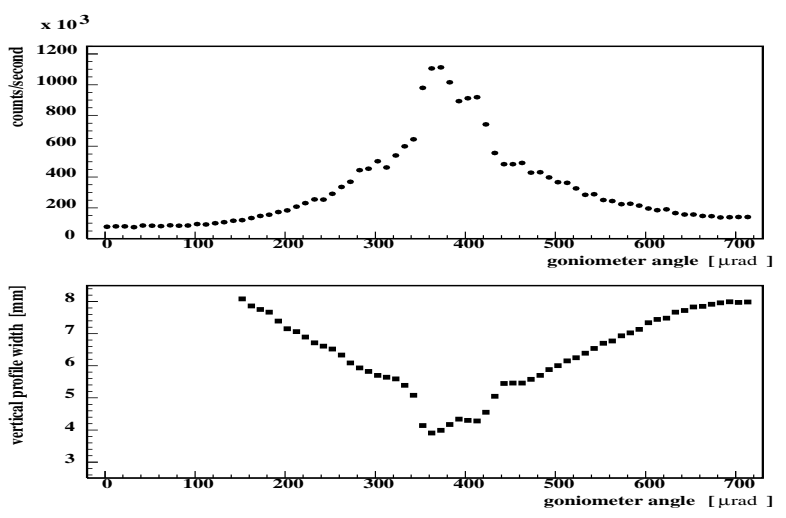

Figure 1: Angular scan and vertical width, $120 \mathrm{GeV}$

Together with the angular scans we show the width of the vertical profiles. It was already demonstrated [1], that the best alignment of the crystal coincides with a minimum of the vertical width of the extracted beam. This is clearly demonstrated for all three energies. The measured widths
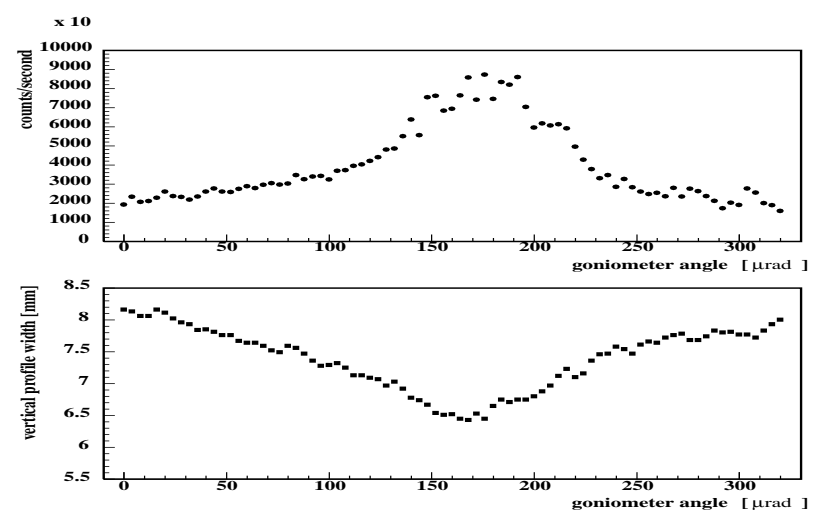

Figure 2: Angular scan and vertical width, $14 \mathrm{GeV}$

of the scans can vary depending on the experimental condition and the type of crystal [1]. This variation is not yet fully understood and is larger than the observable difference between the three energies. The profiles measured with the scintillator hodoscope [1] are shown in Figs. 4 to 6. For $14 \mathrm{GeV}$, a significant widening of the vertical profiles can be observed and is attributed to a stronger multiple scattering of channeled particles at lower energies.

\subsection{Extraction efficiency}

The extraction efficiency should be mainly determined by the probability for channeling and the chance for dechan- 


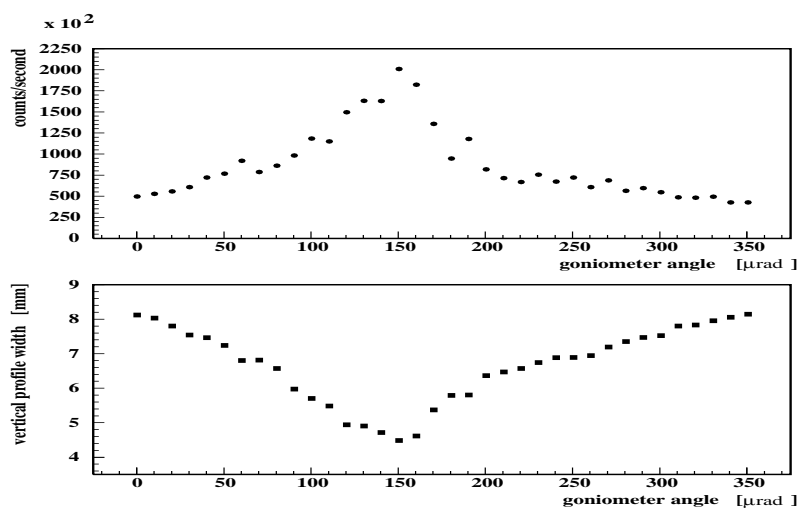

Figure 3: Angular scan and vertical width, $270 \mathrm{GeV}$
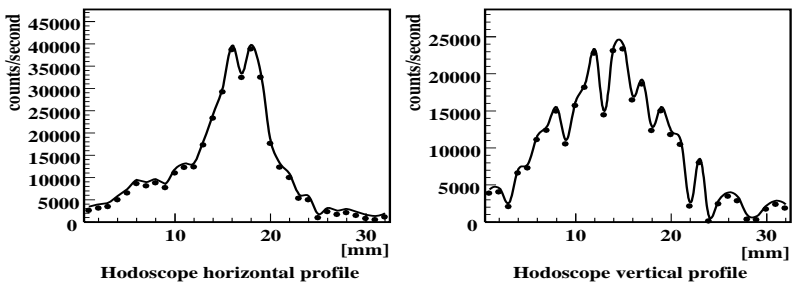

Figure 4: Profiles of extracted beam at $14 \mathrm{GeV}$

neling. We believe that the "channeling probability" is mainly determined by a multipass process. Although we might expect larger impact parameters for $14 \mathrm{GeV}$ particles, the angular scans show no sign of an increased contribution to single pass extraction. For all energies investigated, the multiple scattering in early passes through the crystal is sufficient to increase the impact parameters to values large enough to overcome surface imperfections. Therefore we do not expect the original channeling probability to depend significantly on the energy. The measured widths of the angular scans show also no significantly different behaviour, indicating that the changed critical angle and therefore the angular acceptance play a minor role. Once captured into a channel, the particles can undergo multiple scattering, leading to dechanneling and widening of the profiles. This widening of the profiles should be pronounced in the vertical plane and is clearly observable in Figs. 4 to 6 . The increased dechanneling probability for lower energy should significantly affect the extraction efficiency. We define the efficiency of the extraction process as the ratio of the number of extracted particles to the
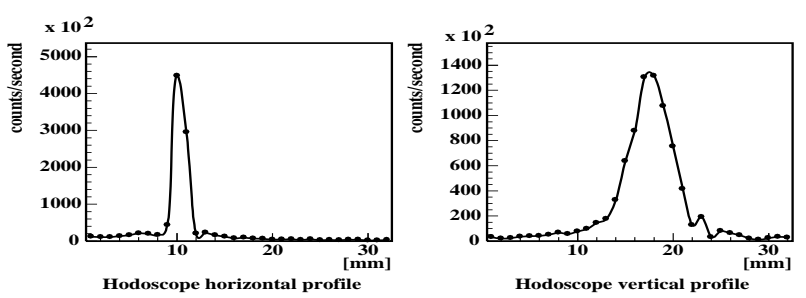

Figure 5: Profiles of extracted beam at $120 \mathrm{GeV}$
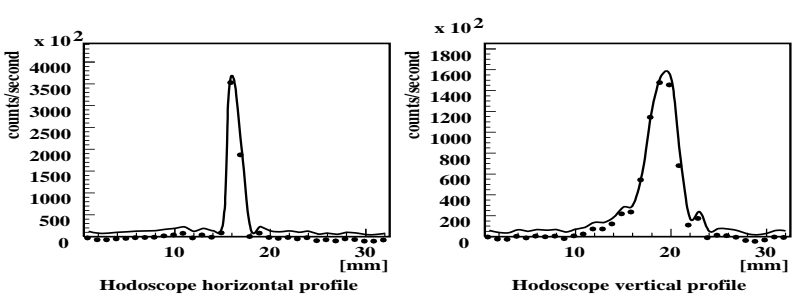

Figure 6: Profiles of extracted beam at $270 \mathrm{GeV}$

number of particles lost from the circulating beam $I_{\text {lost }}$ : $\epsilon_{\text {extr }}=\frac{I_{\text {extr }}}{I_{\text {lost }}}$. The number of extracted particles is determined from a threefold coincidence of trigger scintillation counters and the background from the measured profiles. The number of particles lost from the beam is determined from the beam intensity measured with a beam-currenttransformer (BCT) and the measured beam life time. For the measurement at $14 \mathrm{GeV}$, special care had to be taken for the life time measurement: while for the other energies it was correct to assume an infinite life time without the crystal, the natural life time of the beam at $14 \mathrm{GeV}$ was much lower and only about 0.1 to $4 \mathrm{hrs}$. This natural life time was measured every time and folded into the calculation of the life time reduction due to the crystal. A consequence of the small life time at $14 \mathrm{GeV}$ was that during a measurement the beam intensity changed significantly, thus changing the number of particles intercepting the crystal. This had to be taken into account in the calculation of the efficiency. The result of this analysis is summarized in Tab.1. The ex-

\begin{tabular}{||c|c|c||}
\hline $\begin{array}{c}\text { Beam } \\
\text { energy }(\mathrm{GeV})\end{array}$ & $\begin{array}{c}\text { Extraction } \\
\text { efficiency (\%) }\end{array}$ & $\begin{array}{c}\text { Prediction } \\
\text { simulation (\%) }\end{array}$ \\
\hline 14 & $0.55 \pm 0.3$ & 0.46 \\
\hline 120 & $15.1 \pm 1.2$ & $15.1^{*}$ \\
\hline 270 & $18.6 \pm 2.7$ & 17.7 \\
\hline
\end{tabular}

Table 1: Extraction efficiences at different energies. *Simulation normalized to $120 \mathrm{GeV}$.

traction efficiencies at 120 and $270 \mathrm{GeV}$ are comparable, while the efficiency at $14 \mathrm{GeV}$ is much lower, as expected from qualitative arguments. Also given are the efficiencies predicted from simulation for the three energies. Since the efficiency is usually overestimated in the simulation $[3,5]$ we have normalized it to the value measured at $120 \mathrm{GeV}$ to study the energy dependence and find excellent agreement with the measurement.

\section{REFERENCES}

[1] K. Elsener et al., Nucl.Instr.Meth. B119 (1996) 215.

[2] W. Herr, CERN/SL/92-53 (AP).

[3] G. Vuagnin, CERN/SL/Note 95-24 (AP)

[4] X. Altuna et al., Phys.Lett. B357 (1995) 671.

[5] J. Klem; PhD Thesis, Helsinki Univ. of Technology (1997).

[6] H. Akbari et al., Phys.Lett. B313 (1993) 31. 